\title{
Efecto de los tratamientos antirretrovirales en las manifestaciones orales de los pacientes $\mathrm{VIH}+$
}

\author{
Jané-Salas E*, Chimenos-Küstner E**, López-López J**, Roselló-Llabrés X*, \\ Ocaña-Rivera I***
}

\section{RESUMEN}

El presente trabajo es un estudio para valorar el efecto de los tratamientos antirretrovirales en pacientes seropositivos al VIH, visitados en el dispensario de enfermedades infecciosas del Hospital Vall D’Hebron de Barcelona. Se visitaron 90 pacientes, 51 varones $(56,7 \%)$ y 39 mujeres $(43,3 \%)$, siendo la edad media de los pacientes de 36,2 años con una desviación de $\pm 17,8$ años y una moda de 35 años. Las visitas fueron realizadas desde enero a diciembre del año 1999. Estos pacientes recibían tratamiento antirretroviral de uno o más fármacos y algunos de ellos $(32,2 \%$ ) estaban sometidos a tratamiento antirretroviral de alta actividad (TAAA). Asimismo, en estos pacientes se valoró el recuento de CD4 y carga viral de forma bimodal, al inicio del proceso y en el momento de la visita. La exploración de la cavidad oral se realizó en este momento, buscando las lesiones asociadas al Sida según la clasificación de Pindborg y cols., de 1989.

La patología más prevalente encontrada en este estudio fue la sensación de boca seca o xerostomía en un $47,8 \%$, seguida por orden de frecuencia de policaries $(34,4 \%$ y candidiasis eritematosa $(31,1 \%)$. Otras manifestaciones orales son muy poco frecuentes, en comparación con los resultados de otros autores en la etapa previa a la utilización de los inhibidores de la proteasa (IP). Así, por ejemplo, se observó un solo caso de leucoplasia vellosa, tan característica antes del año 1996.

Palabras clave: VIH, Sida, manifestaciones orales, tratamiento antirretroviral de alta actividad (TAAA).

\section{ABSTRACT}

We evaluate the effects of antiretroviral treatments in HIV seropositive patients, who were seen in the Clinic for Infectious Diseases in the Vall D'Hebron Hospital, Barcelona. 90 patients were seen, 51 males (56.7\%) and 39 females (43.3\%), the mean age of the patients was 36.2 years with a deviation of \pm 17.8 , and a mode of 35 years. The visits were carried out between January and December 1999. These patients were receiving antiretroviral treatment with one or more drugs and some of them $(32.2 \%)$ were subjected to high activity antiretroviral treatment (HAART). Also, in these patients the CD4 count and bimodal viral load was measured, at the start of the disease process and at the time of visit. Theoral cavity was examined at this time, in search of lesions associated with AIDS according to the classification by Pindborg et al, 1989.

The most prevalent pathology found in this study was the sensation of dry mouth or xerostomia in $47.8 \%$, followed by, in order of frequency, multiple caries (34.4\%) and erythematous candidiasis (31.1\%). Other oral manifestations are very rare, in comparison with the results of other authors in the time before the use of protease inhibitors (PI). There is, for example, only one case of leukoplakia, so characteristic before 1996.

Key words: HIV, AIDS, oral manifestations, high activity antiretroviral treatment (HAART).

Aceptado para publicación: Enero 2006. 
* Profesor Asociado de Medicina Bucal, Facultad de Odontología, Universidad de Barcelona.

** Profesor Titular de Medicina Bucal, Facultad de Odontología, Universidad de Barcelona.

*** Profesora Titular de Medicina Interna, Facultad de Medicina (Unidad Vall d'Hebron), Universidad

Autónoma de Barcelona.

Jané-Salas E, Chimenos-Küstner E, López-López J, Roselló-Llabrés X, Ocaña-Rivera I. Efecto de los tratamientos antirretrovirales en las manifestaciones orales de los pacientes VIH+. Av. Odontoestomatol 2006; 22 (6): 315-326.

\section{INTRODUCCIÓN}

En el año 1981 apareció una nueva enfermedad, que afectaba a un tipo de población muy característica y considerada marginal, desde un punto de vista social. Se trataba de los heroinómanos y de los homosexuales. Afectaba secundariamente a otro tipo de pacientes, entre los que se encontraban algunos haitianos y enfermos hemofílicos. Todos ellos tenían en común la aparición de un cuadro clínico caracterizado por la presencia de una inmunosupresión de tipo celular, acompañada de infecciones oportunistas, como la neumonía por Pneumocystis carinii, así como de algunas neoplasias poco frecuentes, como el sarcoma de Kaposi. Es una patología que cumple con el criterio de pandemia (en diciembre de 2005 se calculan 45,3 millones de personas infectadas en el mundo) (1).

Durante estos más de 20 años, sin duda, se han modificado muchos aspectos de esta enfermedad, tanto desde el punto de vista diagnóstico como terapéutico. No es menos cierto que la apariencia clínica, tan característica al inicio, también se ha modificado, debido al diagnóstico precoz y a las terapias antirretrovirales tan efectivas que existen en la actualidad. En el año 1985 se introdujo el primer tratamiento antirretroviral efectivo denominado AZT (zidovudina), que es un inhibidor de la transcriptasa inversa análogo de los nucleósidos. Con este fármaco se inició el control de la enfermedad. A él le siguieron otros inhibidores de la transcriptasa inversa, esta vez no análogos de los nucleósidos. A finales de los años noventa se introdujeron en el arsenal terapéutico los inhibidores de la proteasa, que dieron realmente un cambio importante en el pronóstico de esta enfermedad, al asociarse con los fármacos anteriormente mencionados, surgiendo el concepto de terapéutica antirretroviral de alta actividad (TAAA). Con este tratamiento ha mejorado notablemente el pronóstico de la enfermedad en el primer mundo, modificándose su expresión patológica, también en sus manifestaciones en la cavidad bucal. Antes de la TAAA, la simple observación de la cavidad oral casi permitía un diagnóstico de certeza de la enfermedad e incluso clasificar al paciente en un estadio clínico, que, por supuesto, había que confirmar con las pruebas analíticas pertinentes. Hoy en día se puede sospechar que un paciente, por su historia o sus hábitos, pertenezca a un grupo de riesgo, pero la exploración oral puede ser totalmente anodina. Así, la máxima de que la boca es el termómetro del estadio clínico del sida ha perdido gran parte de vigencia en nuestro ámbito, si bien, por desgracia, en los países en vías de desarrollo, todavía es efectiva (2).

\section{OBJETIVOS}

Los objetivos que se plantearon, para la realización de este trabajo, fueron los siguientes:

1. Realizar un estudio clínico transversal directo en una muestra de pacientes $\mathrm{VIH}$ positivos, de todos los estadios de la enfermedad. En estos pacientes se registrarían la frecuencia de las alteraciones de la cavidad oral que pudieran tener relación con la enfermedad inmune de base.

2. Asociar las frecuencias de las lesiones más comunes dentro de la muestra, con variables demográficas generales como edad, sexo, vías de transmisión asociadas y el tiempo de seropositividad.

3. Valorar los cambios de recuento de CD4 y carga viral en estos pacientes, desde el momento del 
Jané-Salas E, Chimenos-Küstner E, López-López J, Roselló-Llabrés X, Ocaña-Rivera I. Efecto de los tratamientos antirretrovirales en las manifestaciones orales de los pacientes VIH+.

diagnóstico hasta el momento de la exploración estomatológica, así como la terapia antirretroviral asociada.

4. Comprobar si la terapia denominada tratamiento antirretroviral de alta actividad (TAAA) había producido una disminución en la incidencia de la patología oral asociada al sida, en la muestra de pacientes estudiada.

\section{MATERIAL Y MÉTODOS}

\section{Población de estudio}

La población estudiada fue de 90 individuos, de ambos sexos, visitados en el dispensario de consultas externas de la unidad de enfermedades infecciosas del Hospital Vall D’Hebron de Barcelona. Todos ellos habían sido diagnosticados como seropositivos al VIH, entre los años 1986 y 1999. Los pacientes del estudio acudían periódicamente a un control de su patología de base, siendo escogidos al azar por el investigador. En ese momento se tomaban los datos analíticos de esta última visita, así como de la primera de la que se tenía información, para poder procesarlos. Asimismo se aprovechaban estas visitas rutinarias de control, para recoger datos referentes al aparato estomatognático, mediante la exploración sistemática de la cavidad oral.

\section{Recogida de datos}

La recogida de datos la realizó un único examinador, entre enero y diciembre de 1999. Los datos analíticos se obtuvieron de la historia clínica de cada paciente. Posteriormente y de acuerdo con el esquema de Roed-Petersen y Renstrup (3), se procedió a la exploración de la cavidad oral, anejos, así como la zona cervical, en busca de adenomegalias. La xerostomía se valoró como síntoma, preguntando al paciente la sensación subjetiva de sequedad de boca. Mediante una cámara fotográfica Yashica Dental-Eye II se tomaban las fotografías de las manifestaciones clínicas más llamativas o significativas de la patología presente en estos pacientes. Cuando fue conveniente, se solicitó una ortopantomografía por el conducto hospitalario habitual. Los datos recogidos se resumieron en una hoja, siguiendo el protocolo diseñado para este estudio.

\section{Variables}

Entre las variables consideradas, se encuentran unas de carácter cualitativo, categóricas o nominales (sexo, patología, farmacoterapia recibida) y otras de carácter cuantitativo, discretas (no continuas), como son el recuento de CD4 y la carga viral, evaluada en dos momentos diferentes, correspondientes a la primera visita y a la exploración clínica (tablas I-II).

\section{Método de análisis}

Para iniciar el análisis, es de vital importancia conocer si los datos del estudio cumplen o no los criterios de normalidad, para aplicar posteriormente pruebas paramétricas (siguen criterios de normalidad) o no paramétricas (no cumplen criterios de normalidad). Los criterios de normalidad son:

$-N \geq 30$

— Índices de asimetría y curtosis $<2 * \mathrm{DE}$

- Máximo y mínimo dentro del intervalo media \pm 3 DE

Se realiza un análisis de datos apareados (antes-después) para valorar si existen diferencias en la carga viral y en los niveles de CD4 al recibir tratamiento antirretroviral de alta actividad (TAAA). Debido al

\section{TABLA I.- VARIABLES CUALITATIVAS Y CUANTITATIVAS UTILIZADAS}

\begin{tabular}{|l|l|}
\hline Variables cualitativas & Variables cuantitativas \\
\hline $\begin{array}{l}\text { 1. Sexo } \\
\text { 2. Edad }\end{array}$ & 1. Recuento CD4 \\
2. Vara de transmisión & \\
$\begin{array}{l}\text { 4. Terapia antirretroviral } \\
\text { 5. Patología y hábitos } \\
\text { presentes }\end{array}$ & \\
\hline
\end{tabular}




\section{TABLA II.- PATOLOGÍA Y HÁBITOS COSIDERADOS}

1. Boca seca

2. Policaries

3. Candidiasis eritematosa

4. Queilitis angular

5. Leucoplasia vellosa

6. Herpes oral o perioral

7. Leucoplasia retrocomisural

8. Aftosis o úlceras orales

9. Candidiasis pseudomembranosa

10. Patatidomegalia

11. Carcinoma oral

12. Plaquetopenia

13. Sarcoma de Kaposi

14. Hepatitis B

15. Hepatitis C

16. Tuberculosis

17. Consumo de metadona

18. Fotosensibilidad

19. Psoriasis

20. Artralgias

21. Lipodistrofia

22. Tromboflebitis

23. Polineuropatía

24. Diarrea

25. Brote psicótico

26. Ingesta de alcohol

27. Consumo de tabaco

28. Linfoma no Hodgkin

pequeño tamaño muestral se emplean tests no paramétricos. Se utiliza el test de Wilcoxon por ser las variables de estudio (carga viral y niveles de CD4) cuantitativas.

Para la gestión de datos se realizó la depuración de los mismos, no observando valores considerados imposibles, y se aparearon cada uno de ellos según el número de paciente. Para el análisis de los datos se empleó el paquete estadístico SPSS10.0.

\section{RESULTADOS}

\section{Análisis estadístico de los datos}

\section{1) Análisis descriptivo}

El tamaño muestral es de 90 pacientes, siendo un $56,7 \%$ $(n=51)$ hombres y un 43,3\% $(n=39)$ mujeres. La edad media de los pacientes es de 36,2 $\pm 17,8$ años, la moda de 35 años, con un mínimo de 20 y máximo de 72 años. En las mujeres la edad media es de 34,8 $\pm 15,94$ años, la moda de 30 años, con un mínimo de 20 y máximo de 57 años, mientras que en los hombres la edad media es de 37,3 $\pm 18,96$ años, la moda de 35 años, con un mínimo de 22 y máximo de 72 años.

El mecanismo de transmisión de VIH más frecuente es la vía parenteral (ADVP) en un 52,2\% $(n=47)$, seguido de la vía heterosexual con un $30 \%(n=27)$, existiendo diferencias estadísticamente significativas $(p=0,000)$ al estratificar por sexo. En las mujeres observamos que la vía de transmisión de VIH más frecuente es vía heterosexual en un 59\% $(n=23)$, mientras que en los hombres la principal vía es la parenteral (ADVP), en un 66,7\% $(n=34)$.

En la tabla III se observan los fármacos antirretrovirales tomados por nuestros pacientes, siendo la lamivudina (3TC) $(72,5 \%)$ y la estavudina (d4T) $(64,7 \%)$ los más utilizados, seguidos de la zidovudina (AZT) con el 33,3\%. Los tres se sitúan dentro del grupo de análogos de los nucleósidos inhibidores de la transcriptasa inversa, siendo el nelfinavir el inhibidor de la proteasa más utilizado, en el 23,5\% de los pacientes.

Se realizó un estudio comparativo entre sexos y fármacos utilizados por estos pacientes, pero no observamos ninguna diferencia significativa. Era de esperar, ya que la terapéutica se basa en una clasificación clínica bien establecida, en la que el sexo no condiciona la terapia que recibirá el paciente. Sólo en el caso de madres seropositivas gestantes podría valorarse, pero en nuestro grupo de estudio no había ninguna paciente en esta situación.

La patología oral más prevalente (tabla IV) fue la sensación de xerostomía $(47,8 \%, n=43)$, seguida de caries múltiple $(34,4 \%, \mathrm{n}=31)$ y candidiasis eritematosa $(31,1 \%, n=28)$. 


\section{TABLA III.- ANTIRRETROVIRALES UTILIZADOS}

\begin{tabular}{|c|c|c|c|c|c|c|}
\hline & \multicolumn{2}{|c|}{ GLOBAL } & \multicolumn{2}{|c|}{$\sigma^{\pi}$} & \multicolumn{2}{|c|}{ 우 } \\
\hline & $\%$ & n & $\%$ & $\mathbf{n}$ & $\%$ & $\mathbf{n}$ \\
\hline Abacavir & 1,1 & 1 & 2,0 & 1 & 0,0 & 0 \\
\hline Didanosina & 4,4 & 4 & 2,0 & 1 & 7,7 & 3 \\
\hline Lamivudina & 67,8 & 61 & 72,5 & 37 & 61,5 & 24 \\
\hline Estavudina & 62,2 & 56 & 64,7 & 33 & 59,0 & 23 \\
\hline Zalcitabina & 3,3 & 3 & 2,0 & 1 & 5,1 & 2 \\
\hline Zidovudina & 37,8 & 34 & 33,3 & 17 & 43,6 & 17 \\
\hline Tenofovir & 1,1 & 1 & 2,0 & 1 & 0,0 & 0 \\
\hline Efavirenz & 3,3 & 3 & 0,0 & 0 & 7,7 & 3 \\
\hline Nevirapina & 23,3 & 21 & 17,6 & 9 & 30,8 & 12 \\
\hline Indinavir & 16,7 & 15 & 19,6 & 10 & 12,8 & 5 \\
\hline Nelfinavir & 20,0 & 18 & 23,5 & 12 & 15,4 & 6 \\
\hline Ritonavir & 6,7 & 6 & 7,8 & 4 & 5,1 & 2 \\
\hline Saquinavir & 4,4 & 4 & 2,0 & 1 & 7,7 & 3 \\
\hline
\end{tabular}

\section{2) Estudio analítico}

Hay diferencias estadísticamente significativas tanto entre las medias de la carga viral $(p=0,000)$ como en la de los niveles de CD4 $(p=0,001)$ antes y después de recibir tratamiento con un inhibidor nucleósi-

\section{TABLA IV.- PREVALENCIA DE PATOLOGÍA ORAL}

\begin{tabular}{|l|rr|rr|rr|}
\hline & \multicolumn{2}{|c|}{ GLOBAL } & \multicolumn{2}{|c|}{$\boldsymbol{\sigma}^{\mathbf{*}}$} & \multicolumn{2}{|c|}{ \% } \\
\cline { 2 - 7 } & $\mathbf{1}$ & $\mathbf{n}$ & \multicolumn{1}{c|}{$\%$} & $\mathbf{n}$ & $\%$ & $\mathbf{n}$ \\
\hline Xerostomía & 47,8 & 43 & 49 & 25 & 46,2 & 18 \\
$\begin{array}{l}\text { Caries múltiple } \\
\text { Candidiasis }\end{array}$ & 34,4 & 31 & 39,2 & 20 & 28,2 & 11 \\
$\quad$ eritematosa & 31,1 & 28 & 33,3 & 17 & 28,2 & 11 \\
$\begin{array}{l}\text { Queilitis angular } \\
\text { Leucoplasia }\end{array}$ & 2,2 & 2 & 2,0 & 1 & 2,6 & 1 \\
$\quad$ vellosa & 1,1 & 1 & 0,0 & 0 & 2,6 & 1 \\
$\begin{array}{l}\text { Herpes oral } \\
\text { Sarcoma de }\end{array} \quad 4,4$ & 4 & 5,9 & 3 & 2,6 & 1 \\
$\quad$ Kaposi & 3,3 & 3 & 5,9 & 3 & 0,0 & 0 \\
\hline
\end{tabular}

do de la transcriptasa inversa (NRTI). Al estratificar por sexos, estas diferencias se mantienen, excepto los niveles de CD4 en los hombres $(p=0,084)$. No hay diferencias estadísticamente significativas entre las medias de la carga viral $(p=0,172)$ ni en la de los niveles de CD4 $(p=0,069)$ antes y después de recibir tratamiento con un inhibidor de la proteasa, un inhibidor nucleósido de la transcriptasa inversa y un inhibidor no nucleósido de la transcriptasa inversa.

Al estratificar por sexos, en el caso de las mujeres el nivel de significación es de $p=0,461$ para las medias de la carga viral y de $p=0,225$ para los niveles de CD4; por tanto, sin diferencias estadísticamente significativas. Entre los hombres tampoco hay diferencias estadísticamente significativas $(p=0,180$ para la carga viral y $p=0,109$ para los niveles de CD4).

Los niveles de CD4 y la carga viral presentan unas diferencias significativas, en los intervalos de toma de fármacos, en todos los grupos. En el de los ITIAN se establecen unas diferencias estadísticamente significativas, que varían dependiendo del cuartil de edad y sexo que se analicen. Ello también se refleja cuando se añaden al grupo anterior los IP, observándose diferencias estadísticamente significativas tanto entre las medias de la carga viral $(p=0,000)$, como en los niveles de CD4 $(p=0,000)$ antes y después de recibir tratamiento de IP + ITIAN. Al estratificar por sexos, estas diferencias se mantienen, teniendo las mujeres una $\mathrm{p}=0,003$ para los niveles de CD4 y $\mathrm{p}=0,038$ para la carga viral. En los hombres la $p=0,001$ para los niveles de CD4 y $\mathrm{p}=0,001$ para la carga viral. Cuando estudiamos los pacientes que toman la triple terapia IP + ITIAN + ITINN, no se observan diferencias estadísticamente significativas entre las medias de la carga viral $(p=0,172)$, ni en los niveles de CD4 $(p=0,069)$; antes y después de recibir el tratamiento, al estratificar por sexos, estas diferencias se mantienen.

\section{DISCUSIÓN}

Es difícil realizar una comparación acertada y válida entre los diferentes estudios de incidencia y prevalencia de la patología oral asociada a la infección por el VIH, debido a la heterogeneidad epidemiológica, al país de origen del estudio y a la terapéutica que esté recibiendo el paciente. 


\section{TABLA V.- PATOLOGÍA Y NIVELES MEDIOS DE CD4 Y CARGA VIRAL}

\begin{tabular}{|l|ll|cc|}
\hline & \multicolumn{2}{|c|}{ CD4 } & \multicolumn{2}{c|}{ CV } \\
\cline { 2 - 5 } & Pre & Post & Pre & Post \\
\hline Boca seca & 311,02 & 481,51 & 116315,84 & 4430,72 \\
Caries múltiple & 382,53 & 503,86 & 138117,31 & 16157,25 \\
Candidiasis eritematosa & 305,04 & 404,89 & 136205,71 & 51057,85 \\
Queilitis angular & 356,5 & 536 & 13000 & 475 \\
Leucoplasia vellosa & 187 & 400 & 3999 & 8300 \\
Herpes oral & 325,75 & 737,33 & 30249,75 & 443 \\
Plaquetopenia & 722,5 & 967 & 54333,33 & 5917,25 \\
Sarcoma kaposi & 252 & 594 & 753000 & 80325 \\
Hepatitis b & 700 & 313 & - & - \\
Hepatitis c & 412 & 536,93 & 78799,80 & 59521 \\
Tuberculosis & 224 & 240 & 42000 & 6200 \\
Fotosensibilidad & 152,5 & 254 & 3999 & 409150 \\
Psoriasis & 310 & 208 & 320000 & 23000 \\
\hline
\end{tabular}

En una muestra comparable estudiada por Malagón (4) entre los años 1996 y 1998, con 25,7\% de mujeres y $74,3 \%$ de varones, predominaban la transmisión por vía parenteral y la sexual entre los varones homosexuales, aunque la transmisión heterosexual entre las mujeres era la más elevada. Se compara a continuación con algunos estudios de las manifestaciones orales, representativos de la época en que la terapia antirretroviral no incluía los inhibidores de la proteasa (Tabla VI).

Si bien en sus estudios podemos constatar formas afines, las diferencias entre los distintos autores es muy patente, aunque la máxima divergencia la podemos observar entre los grupos americanos y europeos. Las manifestaciones del sarcoma de Kaposi marcan una brecha de difícil explicación, si no tenemos en cuenta la población de estudio. En el caso de Silverman (10), procede casi exclusivamente de pacientes varones cuyo contagio fue por relaciones homosexuales. En todos estos estudios, lo que se constata asimismo es que, independientemente de la presencia de patología específica, la presencia de alguna manifestación oscila entre el 50 y el 55\% en todas las series. En esta comparativa observamos que los datos más concordantes aparecen con el estudio de Chimenos, publicado en 1996 (9). En cuanto a la patología oral más prevalente en el pre- sente estudio (tabla IV y V), la sensación de boca seca, junto con la policaries y la candidiasis eritematosa, son los hallazgos más frecuentes. Respecto al de Malagón (4) sólo se observan coincidencias en el caso de la candidiasis eritematosa, donde la afectación asciende al $50 \%$ en su casuística. También encuentra un $11 \%$ de neoplasias, de las cuales un 56\% son sarcoma de Kaposi. En el presente estudio sólo hemos observado un 3,3\% de neoplasias (3 casos), siendo sarcomas de Kaposi. En el resto de manifestaciones orales no existe coincidencia alguna. Valga como ejemplo la ausencia total de leucoplasias vellosas y 1 caso de leucoplasia retrocomisural $(1,1 \%)$ en nuestro estudio, frente al $25,6 \%$ de leucoplasias vellosas, 3,3\% de leucoplasias de suelo de boca, 43,3\% de leucoqueratosis actínica y 38,3\% de leucoqueratosis nicotínica en el de Malagón, con grupos muestrales comparables. En el presente estudio no se han observado papilomas orales, mientras que suponían un $15,6 \%$ en el grupo de referencia. En nuestro estudio tampoco se diagnosticó ningún caso de lesiones aftosas, frente al 10\% de Malagón.

La diferencia fundamental respecto a otros estudios radica en la utilización de fármacos inhibidores de la proteasa, que se vienen empleando de forma habitual desde el año 1996. Los estudios aparecidos des- 
TABLA VI.- PREVALENCIA DE PATOLOGÍA BUCAL

\begin{tabular}{|c|c|c|c|c|c|c|}
\hline Autor & Candidiasis & $\begin{array}{c}\text { Leucoplasia } \\
\text { vellosa }\end{array}$ & Periodontitis & GUNA & $\begin{array}{c}\text { Sarcoma } \\
\text { de Kaposi }\end{array}$ & $\begin{array}{c}\text { Linfoma no } \\
\text { Hodgkin }\end{array}$ \\
\hline Ceballos, España (5) & $65,7 \%$ & $16,2 \%$ & $78,3 \%$ & & $2,3 \%$ & \\
\hline Phelan, USA (6) & $88 \%$ & $5 \%$ & & & $4 \%$ & \\
\hline Schiodt, Dinamarca (7) & $81 \%$ & $46 \%$ & & & & \\
\hline Laskaris, Grecia (8) & $61 \%$ & $24 \%$ & $19 \%$ & $11 \%$ & $12 \%$ & $2 \%$ \\
\hline Chimenos, España (9) & $33,3 \%$ & $4,76 \%$ & 30,1 & $1,6 \%$ & $3,17 \%$ & \\
\hline Silverman, USA (10) & $70 \%$ & $23 \%$ & & & $45 \%$ & \\
\hline Tukutuku, Zaire (11) & $94 \%$ & $14 \%$ & $17 \%$ & $16 \%$ & $12 \%$ & \\
\hline Porter, Inglaterra (12) & $36,4 \%$ & $15,9 \%$ & $11,4 \%$ & $11,4 \%$ & $2,3 \%$ & \\
\hline Barone, Italia (13) & $53,3 \%$ & $31,3 \%$ & & & $4,6 \%$ & \\
\hline Reichart, Alemania(14) & $54,5 \%$ & $17,3 \%$ & $10,9 \%$ & $5,5 \%$ & $14,5 \%$ & \\
\hline Ramírez, México (15) & $51,2 \%$ & $43,2 \%$ & & & $3,2 \%$ & \\
\hline $\begin{array}{l}\text { Van Der Waal, } \\
\text { Holanda (16) }\end{array}$ & $56 \%$ & & $27 \%$ & & $4 \%$ & \\
\hline Malagón, España (4) & $55 \%$ & $25,6 \%$ & $40,3 \%$ & $2,7 \%$ & $5,5 \%$ & \\
\hline $\begin{array}{l}\text { Khongkunthian, } \\
\text { Tailandia (17) }\end{array}$ & $20,6 \%$ & $11,5 \%$ & & & & \\
\hline Presente estudio & $31,1 \%$ & $1,1 \%$ & & & $3,3 \%$ & \\
\hline
\end{tabular}

de esta fecha inciden en los cambios en la patología puntual o de forma global. Así, existen autores que comentan el descenso de la patología provocada por Candida en los pacientes que reciben este tratamiento (18-20), en combinación con otros antirretrovirales. El mecanismo, según refieren, no está totalmente identificado, aunque se atribuye a la menor carga viral, al aumento de linfocitos CD4 o al aumento de neutrófilos polimorfonucleares circulantes. Lo que sí constata Campisi (21), es que la candidiasis oral es la patología más prevalente en estos pacientes, independientemente de la medicación recibida o de la vía de transmisión.

La presencia de verrugas orales, al contrario de lo que ocurre en otras lesiones, en las series estudiadas aparece como una patología emergente e incluso algunos autores lo constatan como una complicación de la terapia tipo TAAA $(22,23)$. Así Greenwood (24) y Schmidt-Westhausen (25) encuentran una disminución de todas las complicaciones orales, a excepción de las lesiones papilomatosas de la cavidad oral. Esto no ha sido constatado por otros autores (26), ni por nosotros en el presente estudio. Recientes observaciones (27) presentan una posible asociación excluyente en la aparición de leucoplasia vellosa y verrugas orales debido a un déficit local de inmunidad, mediado por el ARN mensajero.

Cuando se trata de las neoplasias oportunistas, la mayoría de autores coinciden en que la TAAA, disminuye la aparición de estos procesos (28-30), e incluso Murdaca (31) indica la posibilidad de tratar a los pacientes con sarcoma de Kaposi, utilizando exclusivamente terapia antirretroviral, no siendo preciso el uso de inhibidores de la proteasa. La presencia de sarcoma de Kaposi no muestra diferencias significativas ( $\mathrm{p}$ $<0.005$ ) en nuestro estudio, comparándolo con otros estudios (32) en que los pacientes no reciben TAAA.

Otros autores coinciden con el presente estudio en que las características poblacionales y el tipo de vía 
de transmisión tienen un factor pronóstico relativo. Lo que realmente predice la evolución del paciente, y secundariamente la incidencia de manifestaciones orales, es la terapia antirretroviral realizada. En concreto, la prescripción de inhibidores de la proteasa, con la conveniente asociación a los antirretrovirales inhibidores análogos o no de los nucleósidos. Así, Patton $(33,34)$, en dos artículos, comenta esta disminución. Es interesante, porque en el primero incide en los cambios analíticos; estos son inversamente proporcionales en el caso del recuento de la carga viral y directamente proporcionales cuando observamos el recuento de CD4. Dentro de este ámbito es importante comentar un trabajo reciente de Greenspan (35), en el que coincide con otros previos (36), en que existe una disminución importante de las infecciones oportunistas, pero llama la atención de los efectos secundarios que pueden presentar estos pacientes, como la distribución anormal de la grasa corporal, la aparición de dislipemia, la intolerancia a la glucosa y otros efectos adversos como un incremento de las formas clásicas de tuberculosis y la aparición de enfermedades poco frecuentes, como la sarcoidosis (37). También hace referencia al incremento de los papilomas orales. Otro factor que destaca, también observado en este estudio, es que la apariencia clínica de las manifestaciones orales ha sufrido tal cambio en nuestro ambiente, que precisa de una exploración mucho más exhaustiva para intentar evidenciar la patología de base que presenta el paciente $(38,39)$.

El recuento de CD4 se ha asociado a la mejoría clínica que presentan los pacientes que reciben TAAA, dato que se refleja en este estudio, así como su asociación con la disminución de la carga viral. Miller (40) asocia esta situación a un mejor pronóstico, no sólo en cuanto a mortalidad, sino a morbilidad.

La restauración de la inmunidad que presentan estos pacientes viene avalada por un incremento de CD4 circulantes. Se asocia a una disminución de la carga viral y como consecuencia a una frecuencia menor de infecciones y patología oportunista, siempre que el paciente tenga una buena adaptación al tratamiento y no surjan resistencias por cepas seleccionadas. Ello cuestiona la necesidad de realizar profilaxis para las enfermedades oportunistas, siempre que los pacientes presenten una situación analítica que no haga sospechar la aparición de una de las situaciones anteriormente comentadas (41-43). Coincidiendo con estos aspectos debe recordarse que estas manifestaciones tendrán su significación dependiendo de la población estudiada y de la terapia realizada. Así en un reciente estudio de Bendick (44), realizado en Camboya, los pacientes estudiados presentaban una prevalencia del $52,5 \%$ de candidiasis pseudomembranosa, 35,6\% leucoplasia vellosa bilateral y un $27,7 \%$ una gingivo-periodontitis ulceronecrótica. Estos pacientes VIH+ no recibían tratamiento antirretroviral alguno. Es motivo de reflexión que estudios realizados contemporáneamente ofrezcan una divergencia tan alta entre los resultados obtenidos (45). En contraste, Cauda y cols. (46) refieren una disminución del $7 \%$ de las candidiasis en los pacientes que toman TAAA, así como prevalencias del $11 \%$ de la leucoplasia vellosa y $1,7 \%$ de gingivoperiodontitis ulceronecróticas. Estos autores no evidencian una diferencia significativa en las otras manifestaciones (aftosis, papilomas, herpes simple y sarcoma de Kaposi). Debe tenerse en cuenta que los pacientes en su estudio tomaban todos ITIAN y la diferencia se estableció al incorporar un inhibidor de la proteasa. Patton (47) afirma, en su casuística, que los papilomas orales aumentan su prevalencia, debido a que los inhibidores de la proteasa no actúan sobre el VPH; también observa un incremento de la patología de las glándulas salivales, con un incremento del $1,8 \%$ al $5 \%$.

En un trabajo posterior, este mismo autor (48) pone de manifiesto y de forma gráfica la variedad de resultados obtenidos, así como la existencia de formas clínicas emergentes en países en vías de desarrollo, como son las infecciones por el hongo Penicilliosis marneffei, en el sudeste asiático, o la aparición de un incremento de noma en África.

Los autores occidentales revisados están de acuerdo en que la mejor manera para realizar el seguimiento de un paciente infectado estriba en realizar controles periódicos de la carga viral y del recuento de CD4. Las lesiones clínicas relacionadas con mala evolución o con progresión rápida de la enfermedad que se referencian son la candidiasis asociada a linfadenopatías cervicales o el crecimiento brusco de las glándulas salivales, pero siempre asociado con una disminución de CD4. Los niveles de carga viral son la otra forma de controlar el proceso de base. 
Jané-Salas E, Chimenos-Küstner E, López-López J, Roselló-Llabrés X, Ocaña-Rivera I. Efecto de los tratamientos antirretrovirales en las manifestaciones orales de los pacientes $\mathrm{VIH}+$.

La utilización de TAAA, también referenciada en los últimos trabajos publicados (49), no deja lugar a dudas en cuanto a que su utilización mejora el pronóstico a largo plazo de estos pacientes. En esta revisión, Reichart, que a su vez cita a Sepkowitz (50), para confirmar sus observaciones, constata también una disminución de la carga viral, así como un incremento de los CD4. Coincide con Greenspan en los efectos adversos observados y añade las interacciones medicamentosas con el astemizol y la terfenadina, que pueden provocar arritmias cardíacas (51).

Trabajos recientes de autores como López (52) y Santos (53) referencian que hay patología de afectación sistémica que puede presentar su primera manifestación en la cavidad oral, coincidiendo con Bascones en esta apreciación (54). Insisten en que los médicos deberían explorar siempre, y a ser posible en primer lugar, la boca de los enfermos de sida.

Baqui (55) buscó la correlación en su estudio de patología periodontal y carga viral. Encontró un incremento de patología periodontal evolutiva cuando la carga viral superaba las 10.000 copias, en comparación con pacientes con menos de 400 copias $/ \mathrm{ml}$.

Otro aspecto encontrado en la literatura (49) es el intento de correlacionar la presencia de alguna lesión específica de la mucosa oral en relación con los diferentes subtipos de VIH, pero no se ha encontrado ningún estudio con cambios significativos que pueda confirmar este hecho.

Recientemente un grupo de trabajo anglo-americano (56) ha intentado unificar los criterios diagnósticos de las lesiones orales que existen en estos pacientes. Además de la identificación clínica de las lesiones, propugnan otros parámetros, como su localización y tamaño. Ello permite realizar comparaciones objetivas entre los diferentes grupos de trabajo, independientemente de la zona geográfica, situación cultural o condición terapéutica que el paciente lleve a cabo. Así, por ejemplo, las úlceras orales se deberían referenciar considerando los siguientes aspectos:

- Tamaño: se puntuará su diámetro en mm, con un máximo de 20 .

- Número: cantidad de úlceras, máximo 20.

- Duración: se cuantifican medias semanas, con un máximo de 10 .
- Tiempo libre de úlceras: semanas libres de lesión, máximo 10.

- Localización: 5, en mucosa no queratinizada y 10 cuando asienta en mucosa queratinizada.

- Dolor: El paciente debe puntuarlo en una escala de 1 a 10.

Dependiendo de la puntuación alcanzada, podrá plantearse una posible pauta de tratamiento e intentar establecer una escala de factor pronóstico. Estos criterios de unificación, aún en fase embrionaria, proporcionarán en un futuro próximo una forma más correcta de comparar los resultados obtenidos por los diferentes grupos de estudio.

Para finalizar, creemos interesante comentar los trabajos de Reichart $(57,58)$, en los que se indica que las manifestaciones patológicas observadas en el tercer mundo, también son subsidiarias de recibir tratamientos antirretrovirales de alta efectividad. Las estadísticas demuestran que la prevalencia de patología es equivalente a la que el llamado primer mundo sufría hace 20 años (59).

\section{CONCLUSIONES}

1. Las manifestaciones orales de estos pacientes, tan comunes antes, son en la actualidad unos hallazgos casuales. En el grupo de pacientes estudiado, la terapia antirretroviral recibida ha demostrado una alta efectividad, que influye en la calidad de vida de estos pacientes, en la disminución de procesos infecciosos oportunistas, tanto de origen bacteriano como vírico, manteniéndose sólo la candidiasis eritematosa como un proceso más prevalente.

2. En nuestro estudio, la vía de contagio, el sexo y el tiempo de seropositividad no han demostrado diferencias significativas en cuanto a la patología oral presente en los pacientes. La presencia de xerostomía se ha presentado con una alta prevalencia, así como las caries y la candidiasis eritematosa. En nuestro estudio no se ha encontrado ninguna manifestación asociada a una vía de transmisión determinada.

3. En el presente estudio el incremento de CD4 y la disminución de la carga viral se relacionan con la toma de TAAA. 
4. La TAAA ha modificado la expresión de las manifestaciones orales que permitían realizar un diagnóstico de sospecha de la seropositividad al VIH o del sida.

\section{BIBLIOGRAFÍA}

1. ONUSIDA. Informe sobre la epidemia mundial VIH/SIDA. Diciembre de 2003. Ginebra; UNAIDS 2003.www.unaids.org URL 1 diciembre 2005.

2. Coogan MM, Greenspan J, Challacombe SJ. Oral lesions in infection with human immunodeficiency virus. Bull World Health Organ. 2005; 83(9):700-6.

3. Roed-Petersen B, Renstrup G. A topographical classification of the oral mucosa suitable for electronic data processing. Its application to 560 leukoplakias. Acta Odontol Scand 1969;27: 681-95.

4. Malagón S. Manifestaciones clínicas de la infección por el virus de la inmunodeficiencia humana en el aparato estomatognático. Tesis Doctoral.Universidad de Barcelona 1998.

5. Ceballos A, Aguirre JM, Bagán JV. Oral manifestations associated with human immunodeficiency virus infection in a Spanish population. J Oral Pathol Med 1996;25:523-26.

6. Phelan JA, Saltzmen BR, Friedland GH, Oral findings in patients with AIDS. Oral Surg Oral Med Oral Pathol 1987;64:50-6.

7. Schiödt M, Pindborg JJ. AIDS and the oral cavity: epidemiology and clinical oral manifestations of HIV infection: a review. Int J Oral Maxillofac Surg. 1987;16:1-14.

8. Laskaris G, Hadjivassiliou M. Oral signs and symptoms in 160 greeks HIV infected patient. J Oral Med 1992;21:120-3.

9. Chimenos E, Malagón SX, Pérez de Rozas M, Caldereny C, Jané E, López J, Roselló X. Prevalencia de patología bucal en pacientes infectados por el VIH. Rev Iberoamericana ETS 1996; 10:99-104.

10. Silverman S Jr, Migliorati CA, Lozada-Nur F, Greenspan D, Conant MA. Oral findings in people with or at high risk for AIDS: a study of 375 homosexual males. J Am Dent Assoc 1986;112:187-92.

11. Tukutuku K, Muyembe-Tamfun L, Kayembe K, Odio W. Oral manifestations of AIDS in a heterosexual population in a Zaire hospital. J Oral Pathol Med 1990;19:232-4.

12. Scully C, Laskaris G, Pindborg J, Porter SR, Reichart P. Oral manifestations of HIV infection and their management. I. More common lesions. Oral Surg Oral Med Oral Pathol 1991;71:158-66.

13. Ficarra G, Barone R, Gaglioti D. Oral hairy leukoplakia amonh HIV+ intravenous drug abusers: a clinicopathologic and ultraestructural study. Oral Surg Oral Med Oral Pathol Oral Radiol Endod 1995;79:570-1.

14. Reichart PA. Infections of the oral mucosa II.Bacterial, mycotic and viral infections. Mund Kiefer Gesichtschir 1999;3:298-308.

15. Ramírez V, González A, De la Rosa E, González M. Oral lesions in Mexican HIV infected patients. Oral Pathol Med 1990;19:482-5.

16. Van der Waal I, Schulten EA, Pindborg JJ. Oral manifestations of AIDS: an overview. Int Dent $\mathrm{J}$ 1991;41:3-8.

17. Khongkunthian P, Grote M, Isaratanan W, Plyaworawong S, Reichart PA. Oral manifestations in HIV-positive adults from Northern Thailand. J Oral Pathol Med 2001;30:220-3.

18. Hoegl L, Thoma-Greber E, Rocken M, Korting HC. HIV protease inhibitors influence the prevalence of oral candidosis in HIV-infected patients: a 2-year study. Mycoses 1998;41:321-5.

19. Diz Dios P, Ocampo A, Miralles C, Otero I, Iglesias I, Rayo N. Frequency of oropharyngeal candidiasis in HIV-infected patients on portease inhibitor therapy. Oral Surg Oral Med Oral Pathol Oral Radiol Endod 1999;87:437-41.

20. Amador C, Bodi V, Pasquau F, Ena J, Benito C, Fenoll VF. Factores de riesgo asociados a la aparición de infecciones durante el primer año de tratamiento antiretroviral de alta eficacia. Med Clin (Barc) 2001;116:41-6. 
Jané-Salas E, Chimenos-Küstner E, López-López J, Roselló-Llabrés X, Ocaña-Rivera I. Efecto de los tratamientos antirretrovirales en las manifestaciones orales de los pacientes $\mathrm{VIH}+$.

21. Campisi G, Pizzo G, Mancuso S, Margiotta V. Gender differences in human immunodeficiency virus-related oral lesions: an Italian study. Oral Surg Oral Med Oral Pathol Oral Radiol Endod 2001;92:546-51.

22. Greenspan D, Canchola AJ, MacPhail LA, Cheikh B, Greenspan JS. Effect of highly antiretroviral therapy on frequency of oral warts. Lancet 2001; 357: 1411-2.

23. King MD, Reznik DA, O’Daniens CM, Larsen NM, Osterholt D, Blumberg HM. Human papillomavirus-associated oral warts among human immunodeficiency virus-seropositive patients in the era of highly active antiretroviral therapy: an emerging infection. Clin Infect Dis 2002;34:641-8.

24. Greenwood I, Zakrzewska JM, Robinson PG. Changes in the prevalence of HIV-associated mucosal disease at a dedicated clinic over 7 years. Oral Dis 2002;8:90-4.

25. Schmidt-Westhausen AM, Priepke F, Bergmann FJ, Reichart PA. Decline in the rate of oral opportunistic infections following introduction of highly active antiretroviral therapy. J Oral Pathol Med 2000; 29: 336-41.

26. Ceballos A, Gaitán LA, Ceballos L, Lezama D. Oral lesions in HIV/AIDS patients undergoing highly active antiretroviral treatment including protease inhibitors: a new face of oral AIDS?. AIDS Patient Care 2000; 14: 627-35.

27. Lilly EA, Cameron JE, Shetty KV, Leigh JE, Hager S, McNulty KM, Cheeks C, Hagensee ME, Fidel PL Jr. Lack of evidence for local immune activity in oral hairy leukoplakia and oral wart lesions. Oral Microbiol Immunol. 2005 ;20(3):154-62.

28. Tam HK, Zhang ZF, Jacobson LP, Margolick JB, Chmiel S, Rinaldo C, Detels R. Effect of highly active antiretroviral therapy on survival among HIVinfected men with Kaposi's sarcoma or non-Hodgkin lymphoma. Int J Cancer 2002; 98: 916-22.

29. Kirk O, Pedersen C, Cozzi-Lepri A, Antunes F, Miller V, Gatell JM, Katlama C, Lazzarin A, Skinhoj P, Barton SE. Non-Hodgkin lymphoma in HIVinfected patients in the era of highly active antiretroviral therapy. Blood 2001;98:3406-12.
30. Cortes J, Thomas D, Rios A, Koller C, O’Brien S, Jeha S, Faderl S, Kantarjian H. Hyperfractionated cyclophosphamide, vincristine, doxorubicin, and dexamethasone and highly active antiretroviral therapy for patients with acquired immunodeficiency síndrome-related Burkitt lymphoma/leukemia. Cancer 2002; 94: 1492-9.

31. Murdaca G, Campelli A, Setti M, Indiveri F, Puppo F. Complete remission of AIDS/Kaposi's sarcoma after treatment with a combination of two nucleoside reverse transcriptase inhibitors and one non-nucleoside reverse transcriptase inhibitor. AIDS 2002;16:304-5.

32. Schmidt-Westhausen AM, Priepke F, Bergmann FJ, Reichart PA. Decline in the rate of oral opportunistic infections following introduction of highly active antiretroviral therapy. J Oral Pathol Med 2000; 29: 336-41.

33. Patton LL. Sensitivity, specificity, and positive predictive value of oral opportunistic infections in adults with HIV/AIDS as markers of immune suppression and viral burden. Oral Surg Oral Med Oral Pathol Oral Radiol Endod 2000;90:182-8.

34. Patton LL, McKaig R, Strauss R, Rogers D, Eron JJ Jr. Changing prevalence of oral manifestations of human immune-deficiency virus in the era of protease inhibitor therapy. Oral Surg Oral Med Oral Pathol Oral Radiol Endod 2000;89:299-304.

35. Greenspan S, Greenspan D. The epidemiology of the oral lesions of HIV infection in the developed world. Oral Dis 2002; 8: 34-9.

36. Aguirre JM, Echebarria MA, Ocina E, Ribacoba L, Montejo M. Reduction of HIV-associated oral lesions after highly active antiretroviral therapy. Oral Surg Oral Med Oral Pathol Oral Radiol Endod 1999; 88:114-5.

37. Mirmirani P, Maurer TA, Herndier B, McGrath M, Weinstein MD, Berger TG. Sarcoidosis in a patient with AIDS: a manifestation of immune restoration syndrome. A Am Acad Dermatol 1999;41:285-6.

38. Cruz G, Lamster I. The accurate diagnosis of oral lesions in HIV infection: impact on medical staging. Arch Otolaryng Head Neck Surg 1996; 122:68-73. 
39. Paauw DS, Wenrich MD, Curtis R. Ability of primary care physicians to recognize physical findings associated with HIV infection. JAMA 1995; 274:1380-2.

40. Miller V, Sabin CA, Phillips AN, Rottmann C, Rabenau H, Weidmann E, Rickerts V, Findhammer S, Helm EB, Staszewski S. The impact of protease inhibitor-containing highly active antiretroviral therapy on progression of HIV disease and its relationship to CD4 and viral load. AIDS 2000;14:2129-36.

41. Powderly WG. Long-term exposure to lifelong therapies. J Acquir Inmune Defic Syndr 2002: 29: S28-40.

42. Lederman MM, Valdez H. Immune restoration with antiretroviral therapies: implications for clinical management. JAMA 2000; 284:223-8.

43. Powderly WG. Prophylaxis for opportunistic infections in an era of effective antiretroviral therapy. Clin Infect Dis 2000; 31:597-601.

44. Bendick C. Oral manifestations in Cambodians with HIV and AIDS. J Oral Pathol Med 2002; 31:1-4.

45. Morgan D, Mahe C, Mayanja B, Whitworth J. Progression to symptomatic disease in people infected with HIV-1 in rural Uganda: prospective cohort study. BMJ 2002;324:193-7.

46. Cauda R, Tacconelli E, Tumbarello M. Role of protease inhibitors in preventing recurrent oral candidosis in patients with HIV-infection: a prospective case-control study. J AIDS 1999;21:20-5.

47. Patton LL, McKaig R, Strauss R, Rogers D, Eron JJ Jr. Changing prevalence of oral manifestations of human immune-deficiency virus in the era of protease inhibitor therapy. Oral Surg Oral Med Oral Pathol Oral Radiol Endod 2000; 89:299-304.

48. Patton LL, Phelan Ja, Ramos-Gómez FJ, Nittayananta W, Shiboski CH, Mbuguye TL. Prevalence and classification of HIV-associated oral lesions. Oral Dis 2002;8:98-109.

49. Birnbaum W, Hodgson TA, Reichart PA, Sherson W, Nittayannanta SW, Axell TE. Prognostic significance of HIV-associated oral lesions and their relation to therapy. Oral Dis 2002;8:110-4.
50. Sepkowitz KA. Effect of HAART on natural history of AIDS-related opportunistic disorders. Lancet 1998;104:559-64.

51. Porter SR, Scully C. HIV topic update: protease inhibitor therapy and oral health care: Oral Dis 1998;4:159-63

52. López DB, Sambuelli R, Femopase F. Bacillary angiomatosis affecting the oral cavity. Report of two cases and review. J Oral Pathol Med 2000; 29:91-3.

53. Santos R, Cardoso O, Rodrigues P. Bacillary angiomatosis by Bartonella quintana in an HIVinfected patient. J Am Acad Dermatol 2000;42: 299-301.

54. Bascones A, Serrano C, Campo J. Oral manifestations of HIV infection. Med Clin (Barc). 2003 Mar 29;120(11):426-34.

55. Baqui AAMA, Meiller TF, Jabra-Rizk MA. Association of HIV viral load with oral diseases. Oral Dis 1999;5:294-8.

56. Flint S, Glick M, Patton L, Tappuni A, Shirlaw P, Robinson P. Consensus guidelines on quantifying HIV-related oral mucosal disease. Oral Dis 2002; 8:115-9.

57. Reichart PA, Khongkhunthian P, Bendick C. Oral manifestations in HIV-infected individuals from Thailand and Cambodia. Med Microbiol Immunol (Berl). 2003 Aug;192(3):157-60. Epub 2003 Jan 18.

58. Reichart PA. Oral manifestations in HIV infection: fungal and bacterial infections, Kaposi's sarcoma. Med Microbiol Immunol (Berl). 2003 Aug;192(3):165-9. Epub 2003 Mar 05.

59. Patton LL. HIV disease.Dent Clin North Am. 2003 Jul;47(3):467-92.

\section{CORRESPONDENCIA}

Dr. Enric Jané Salas

Viladomat 110-112

08015 - Barcelona (España)

e-mail: enrijane@menta.net 